\title{
El consentimiento informado en las publicaciones latinoamericanas de Psicología
}

\section{0 consentimento informado nas publicações latino-americanas de Psicologia Informed Consent in Latin American Psychological Publications}

\author{
Gina Chávez Ventura* \\ Universidad Privada del Norte, Perú \\ Henry Santa Cruz Espinoza \\ Universidad César Vallejo, Perú \\ Mirian Pilar Grimaldo Muchotrigo \\ Universidad Peruana de Ciencias Aplicadas, Perú \\ Doi: dx.doi.org/10.12804/ap132.2.2014.12
}

\section{Resumen}

El propósito fue determinar si las publicaciones psicológicas incluyen el consentimiento informado en el procedimiento de sus investigaciones. Se analizaron 1924 artículos de investigaciones empíricas, de ocho países de América Latina, publicados entre los años 1994 y 2012 en revistas indexadas en el catálogo de Latindex. La obtención del consentimiento informado se incrementó muy irregularmente en el año 2012; sin embargo, hay un alto porcentaje de publicaciones que no lo obtuvieron. Los países que contemplan el consentimiento informado en sus códigos de ética tienden a su mayor aplicación. Consecuentemente, no solo es necesario normar el consentimiento informado sino también promover la reflexión crítica de la dimensión científica de la Psicología en un marco deontológico y ético, desde la formación profesional.

Palabras clave: Consentimiento informado, deontología, publicaciones

\section{Resumo}

O propósito foi determinar se as publicações psicológicas incluem o consentimento informado no procedimento de suas pesquisas. Analisaram-se 1924 artigos de pesquisas empíricas de oito países da Latino-América, publicados entre os anos 1994 e 2012 em revistas indexadas no catálogo de Latindex. A obtenção do consentimento informado esteve se incrementando muito irregularmente ao ano 2012; no entanto, existe uma alta porcentagem de publicações que não obtiveram consentimento. Os países que contemplam o consentimento informado em seus códigos de ética tendem a sua maior aplicação. Consequentemente, não só é necessário regular o consentimento informado mas também promover a reflexão crítica da dimensão científica da Psicologia em um marco deontológico e ético, desde a formação profissional.

Palavras-chave: Consentimento informado, deontologia, publicações

\footnotetext{
* Gina Chávez Ventura, Facultad de Ciencias de la Salud, Universidad Privada del Norte; Henry Santa Cruz Espinoza, Escuela Profesional de Psicología, Facultad de Humanidades, Universidad César Vallejo; Mirian Pilar Grimaldo Muchotrigo, Facultad de Ciencias Humanas, Área de Investigación de la Dirección de Calidad Educativa, Universidad Peruana de Ciencias Aplicadas.

La correspondencia relacionada con este artículo debe ser dirigida a Gina Chávez Ventura, Calle Las Casuarinas 280. Departamento 302. Urb. Santa Edelmira. Distrito Víctor Larco. Trujillo, Perú. Correo electrónico: gina.chavezv@hotmail.com
}

Para citar este artículo: Chávez, V. G., Santa Cruz, E. H. \& Grimaldo, M. M. (2014). El consentimiento informado en las publicaciones latinoamericanas de psicología. Avances en Psicología Latinoamericana, 32(2), 345-359. doi: dx.doi.org/10.12804/apl32.2.2014.12 
fbstract

The purpose was to determine if the psychological publications included the informed consent in the procedure of their research. 1924 articles of empirical research from eight Latin American countries published between 1994 and 2012 in journals indexed in the Latindex catalogue were analyzed. It's reported that the obtaining of informed consent has increased in an irregular way in 2012; however, there is a high percentage of publications that did not obtain it. The countries that considered the informed consent in their codes of ethics tend to have the highest rate of application. Consequently, it's not only necessary to establish norms for informed consent but also to promote the critical reflection of the scientific dimension of psychology within an ethical framework, starting with professional training.

Keywords: Informed consent, ethics, publications

Toda ciencia aspira a su adecuado desarrollo y para ello debe establecer una estrecha relación con la filosofía y deberá definir con claridad las esferas que esta comprende, como son la dimensión lógica (el lenguaje matemático), la dimensión gnoseológica (teoría del conocimiento y lo metodológico), la dimensión semántica (la definición de sus conceptos), la dimensión ontológica (concepción del ser humano y su naturaleza) y la dimensión ética (obrar bien) (Bunge \& Ardila, 2002).

La dimensión ética establece los límites a la acción del investigador, lo que se constituye en una necesidad imperante al trabajar con seres humanos. La bioética así lo propugna en la defensa del respeto y la promoción de la dignidad humana dentro del ámbito biomédico y de la atención en salud (León, 2011), mediante el análisis y reflexión a la luz de valores y principios morales (Ciccone, 2006; Lucas, 2010).

El concepto de bioética fue acuñado por Potter (1970) con la intención de integrar los tradicionalmente escindidos hechos biológicos y los valores éticos y velar por la supervivencia humana y la mejora de la calidad de vida, ante el riesgo de la deshumanización por el progreso científico, los avances científicos y técnicos de la medicina moderna que ampliaron considerablemente el modo de intervenir sobre el paciente y ejercer un amplio control sobre él (León, 2011; Ruiz \& Marijuán, 2009), así como por los problemas de investigación ocurridos con los seres humanos (León, 2011).

Los planteamientos eugenésicos propiciaron el genocidio y experimentos médicos en los campos de concentración nazi (Ruiz \& Marijuán, 2009), cuyas consecuencias dieron lugar en el año 1948 al Código de Núremberg, donde se abordó en un primer plano el problema del consentimiento voluntario en las investigaciones con humanos (Mainetti, 1989), y se utilizó para juzgar a los médicos nazis que experimentaron con prisioneros en los campos de concentración. Si bien esta normatividad ha sido aplicada inicialmente en el ámbito médico, marca un hito en la consideración de la ética en la investigación científica, especialmente experimental. Es en 1957, en un juicio del caso Salgo en California (EEUU), cuando la sentencia incluye los términos consentimiento informado (Anaya, 2008) para referirse al derecho de los pacientes a recibir información sobre los procedimientos médicos a los que van a someterse y decidir libre y voluntariamente si lo desean o no (Ruiz \& Marijuán, 2009).

También van surgiendo una serie de declaraciones y normas. Así, en 1948 se promulgó la Declaración internacional de los derechos humanos de la Organización de las Naciones Unidas (Naciones Unidas, s.a.), donde se reconoce que los seres humanos nacen libres e iguales en libertad y derechos. En ese mismo año, en la Declaración de Ginebra se explicita el compromiso del médico de velar por la salud del paciente (Asociación Médica Mundial, 2002; Chavarría, 2009).

En 1974, el Congreso norteamericano constituyó la National Commission for the Protection of Human Subjects of Biomedical and Behavioral Research y tras cuatro años de trabajo formuló tres principios éticos en el Informe Belmont (1978). Entre ellos se encontraban la beneficencia (no producir daños, orientado a maximizar ventajas y minimizar riesgos), la autonomía (respeto de la voluntad) y la justicia, que también son valorados en el campo psicológico por França-Tarragó (1996) como principios psicoéticos.

Si bien es cierto que la bioética ha emergido desde el campo de la medicina, la psicoética se desprende de ella. Según França-Tarragó (1996) esta 
última se refiere a los dilemas éticos de la relación entre pacientes y profesionales de la salud mental. La psicoética busca orientar la intención y el fin de las investigaciones centradas en el deber del profesional y en los derechos de los participantes, no solo para evitar incurrir en faltas de investigaciones como las ya efectuadas (e.g. estudios de Festinger en 1957 sobre disonancia cognitiva, o el de Milgran sobre obediencia a la autoridad publicado en 1963 o el experimento de Zimbardo, Haney, Curtis Banks \& Jaffe en 1971 sobre el encarcelamiento; o el experimento para producir tartamudez realizado en 1939 por Tudor), sino para buscar el máximo bienestar de quienes participan en los estudios.

La preocupación por la conducta ética de los psicólogos llevó a la American Psychological Association (APA) en el año 1992 a publicar un código de conducta, con la finalidad de guiar las acciones de los psicólogos hacia el cumplimiento de los siguientes principios éticos: preocupación por el bienestar de los demás, responsabilidad profesional y científica, integridad, respeto por los derechos y dignidad humana. Sin embargo, en el año 2002 algunos de dichos principios fueron afinados (principio de beneficencia y no maleficencia, fidelidad y responsabilidad y respeto a los derechos y la dignidad humana), el de justicia fue incluido y el principio de integridad se mantuvo (APA, 2002). Estos imperativos categóricos se han mantenido vigentes al año 2010 (APA, 2010a) y engloban los principios referidos en el Informe Belmont (1978).

Además, los integrantes de la Comisión ética conformada por la Federación de Psicólogos de la República Argentina, Colegio de Psicólogos de Bolivia, Conselho Federal de Psicología de la República Federativa del Brasil, Colegio de Psicólogos de Chile, Sociedad Paraguaya de Psicología y Coordinadora de Psicólogos del Uruguay, en noviembre de 1997 firmaron el protocolo de acuerdo marco de principios éticos para el ejercicio profesional de los psicólogos en el Mercosur y países asociados: respeto de los derechos y dignidad de las personas, competencia, compromiso profesional y científico, integridad y responsabilidad social (Hermosilla, 2000). Estos principios también han sido adoptados por la Asamblea de la Unión Internacional de Ciencia Psicológica (International
Union of Psychological Science, 2008) en la Declaración universal de principios éticos para psicólogas y psicólogos con la finalidad de disponer de un fundamento común para desarrollar y evaluar los códigos de ética específicos de cada región o país. El principio de compromiso profesional y científico de los psicólogos de Mercosur, por definición, se encuentra incluido en el principio responsabilidades profesionales y científicas con la sociedad, referido por la Asamblea de la Unión Internacional de Ciencia Psicológica.

Así también, la declaración de principios de la Unión Latinoamericana de Entidades de Psicología (ULAPSI, s.a.), señala que una de sus finalidades es fomentar el desarrollo y la intervención de prácticas psicológicas éticas.

Garantizar el cumplimiento de los principios éticos en el terreno de la investigación científica, implica la obtención del consentimiento informado, entendido como la autorización para participar de la acción profesional, luego de comprender la información, valorar las alternativas y tomar una decisión razonada respecto de lo que es más conveniente o no para el o la participante, apoyado en su facultad de elegir libremente, sin coacción ni interna ni externa (Calva, 2006; Leibovich, 2000; Vélez, 1996).

El consentimiento informado implica no solo velar por el bienestar del paciente, usuario o participante de la investigación, sino considerar su libre determinación o autonomía. Para Acevedo (2002), la autonomía se cumple cuando existe una ausencia de control externo, intencionalidad y conocimiento.

En 1998, França et al. referían que el consentimiento válido debe caracterizarse por ser veraz, libre, oportuno, formal e interactivo. De manera similar, la APA (2010b) considera que para obtener el consentimiento informado, los psicólogos tienen el deber de informar a los participantes el propósito de la investigación, la duración esperada y los procedimientos; su derecho de retirarse del estudio aun cuando ya se haya iniciado, las consecuencias esperables de declinar del estudio; los factores que puedan influir en la decisión de participar como posibles riesgos y efectos adversos; los beneficios esperados, los límites de la confidencialidad; los incentivos de la participación y a quién contactar 
para responder a las preguntas relacionadas con la investigación. Si el trabajo fuera experimental, es deber informar los procedimientos experimentales, tratamientos alternativos si el participante decide retirarse del estudio y servicios disponibles para el grupo control.

Algunos autores (Figueroa, 1999; Lolas, 2002) coinciden en señalar que el consentimiento informado es un proceso que no puede reducirse solo a la firma de un formato que indique la aceptación de la participación. Tampoco se puede considerar como un formato único que sirve para diversas investigaciones. De allí la necesidad de redactarlo en función a la naturaleza y los objetivos de la investigación, sin perder de vista el respeto por los participantes. Es por ello importante que el participante no solo lea el consentimiento informado, sino que lo comprenda en términos de sus derechos como persona investigada (Mann, 1994). Ello implica que los investigadores conozcan cuáles son las perspectivas de los participantes, lo cual significa comprender sus expectativas, preocupaciones y creencias acerca de su participación (Richaud, 2007).

Algunos códigos de ética del psicólogo explicitan la obtención del consentimiento informado dentro de sus normas o pautas de conducta, así están el Colegio de Psicólogos de Chile (1999), la Federación de Psicólogos de la República Argentina (1999), la Coordinadora de Psicólogos del Uruguay (Coordinadora de Psicólogos del Uruguay, Sociedad de Psicología del Uruguay, Facultad de Psicología, UDELAR y Facultad de Psicología, UCU, 2001), la Asociación de psicólogos de Puerto Rico (2007), la Sociedad Paraguaya de Psicología (2012) y el Colegio de Psicólogos de Cochabamba (1995). En otros códigos, como los de la Sociedad Mexicana de Psicología (2007), el Código deontológico y bioético para el ejercicio de la Psicología en Colombia (Ley $N^{\circ} 1090$ de 2006), el de la Federación de Psicólogos de Venezuela (1981) y el Colegio de Psicólogos del Perú (1981), se incluyen detalles del consentimiento (no lo denominan consentimiento informado), mientras que en los códigos del Colegio Profesional de Psicología de Costa Rica (2010), del Colegio de Psicólogos de la provincia de Santa Cruz (Bolivia) y el Conselho Federal de Psicologia (2005) del Brasil se mencio- na el consentimiento, pero no se describe el procedimiento de su obtención.

Existen, por tanto, variaciones entre el marco normativo del psicólogo entre los diferentes países latinoamericanos; sin embargo, el manual de publicaciones de la APA (2010b) sí contempla el proceso de obtención del consentimiento informado, de manera detallada, señalando incluso sus excepciones.

La adhesión a los códigos de ética y a las normas de la APA - que regulan el ejercicio profesional y la investigación, respectivamente- legitima la praxis profesional del psicólogo y responde a las expectativas sociales.

Como bien señala Sanz (2008), las acciones que realice el profesional de la psicología exigen una reflexión crítica de los saberes teóricos, instrumentales y axiológicos, ya que es indudable que el psicólogo, al poseer un contexto e historia personal, pueda tener perspectivas distintas de lo bueno que se contraponen con sus clientes o usuarios, por ello es necesario ofrecer una explicación de los procedimientos - profesionales y científicos- posibles a emplear en términos adecuados y apropiados a la cultura de los participantes, de tal modo que sean ellos quienes decidan lo que es bueno o no para sí mismos. Además, como indica Rodríguez (2005), la educación de quienes llevan a cabo las investigaciones es fundamental para que tengan conciencia crítica y ética, además la conducta ética de la investigación se ve fortalecida por el contexto sociopolítico y cultural de cada país.

Ante ello, es de interés lograr la interiorización de los principios ya referidos en un desempeño ético, profesional y científico del psicólogo. En consecuencia, la labor ha de iniciarse en las aulas universitarias. Al respecto, Martínez, Buxarrais y Bara (2002) señalan tres maneras para integrar la dimensión ética en la formación universitaria: (a) la formación deontológica del estudiante como futuro profesional, (b) la formación deontológica de los docentes y en su función de modelo susceptible de aprendizaje social por parte de los estudiantes; y (c) la formación ética del estudiante.

La necesidad de considerar la dimensión ética en la formación profesional se sustenta en los hallazgos de las investigaciones. Así, en los estudios encontrados se reporta que el consentimiento in- 
formado evita posibles riesgos para el usuario de los servicios médicos, gracias a la confianza social y expectativas que atribuye al profesional (Eval, 2012). Incluso, en investigaciones realizadas en la web, es importante proteger a los participantes y garantizar la confidencialidad y para ello los Comités de ética desempeñan un rol protagónico (Mc Rae, 2011; Vayena, Mastroianni \& Kahn, 2012).

Otras investigaciones coinciden en afirmar el déficit formativo de temas éticos en los profesionales de psicología de Mar del Plata (Hermosilla, Liberatore, Losada, Della Savia \& Zanatta, 2006) y en los estudiantes de psicología del último año de una universidad argentina (Sánchez, 2010). También el análisis de informes de ensayos aleatorizados en racimo (CRT) publicados en MEDLINE por Giraudeau, Caille, Gouge y Ravaud (2012) reportó que la notificación de la aprobación del comité de ética y el consentimiento informado participante es menor al óptimo. A pesar de ello, el estudio de Pease-Carter y Minton (2012) encontró que estudiantes de maestría de programas de counseling valoraron el consentimiento informado con moderada a extrema importancia.

A pesar de las investigaciones referidas, son escasos los estudios acerca de variables que pueden asociarse al consentimiento informado. Al respecto Supady, Antonie, Joachim, Udo y Northoff(2011), en su estudio exploratorio con hombres sanos y pacientes psiquiátricos forenses y no forenses, hallaron que una mayor empatía cognitiva y reconocimiento de emociones positivas se asociaron con una mayor capacidad de decisión y mayores tasas de negativa a dar su consentimiento informado.

El estado del arte refleja el vacío de la dimensión ética -a pesar de su importancia- en la formación y ejercicio profesional del psicólogo, lo que lleva a plantear como interrogante si dicha falencia se presenta también en el quehacer investigativo, entendiendo que la investigación no solo debe responder (Flores, 2010) a las demandas planteadas por la sociedad; sino que debe garantizar el máximo bienestar de los participantes basado en su libre albedrío. Por ello, es preciso reflexionar si las investigaciones publicadas en revistas acreditadas de la región latinoamericana, se apoya en estándares éticos. De esta manera, se pretende dar respuesta a la pregunta ¿en qué medida las investigaciones empíricas psicológicas latinoamericanas publicadas en revistas indexadas en el Catálogo de Latindex incluyen en la descripción de su procedimiento el consentimiento informado?

El problema se estudia desde una perspectiva bibliométrica para ofrecer un panorama del statu quo de las consideraciones éticas en el desarrollo de la investigación científica y promover la práctica de la obtención del consentimiento informado en estudiantes y profesionales de la psicología.

Se han propuesto como objetivos determinar el porcentaje de obtención del consentimiento informado en las publicaciones en revistas de psicología de los países Perú, Colombia, México, Puerto Rico, Argentina, Chile, Brasil, Costa Rica, Paraguay, Uruguay, Bolivia y Venezuela, desde el año 1994 hasta el año 2012 y determinar la frecuencia de obtención del consentimiento informado en las investigaciones experimentales publicadas por los países referidos entre los años 1994 y 2012.

\section{Método}

Se realizó el análisis de un total de 1924 artículos de investigación empírica publicados en revistas de psicología indexadas en el catálogo de Latindex. Se recolectaron las publicaciones desde el año 1994 hasta el mes de noviembre del 2012, disponibles en la web a texto completo (Tabla 1), de Perú, Colombia, Chile, México, Costa Rica, Argentina, Puerto Rico, Brasil, Bolivia, Paraguay, Uruguay y Venezuela. Se consideró desde 1994, ya que en ese año hubo más de un país que publicó artículos empíricos en revistas de psicología y con ello es posible hacer una comparación entre los países.

Por no reunir los criterios de inclusión, adelante referidos, no fueron incluidos Ecuador, El Salvador, Guatemala, Honduras, Nicaragua, Panamá y República Dominicana. Tampoco se incluyó Cuba porque la única revista de psicología indexada en Latindex dejó de publicar en el año 2003.

Para la investigación no se tomaron en cuenta los trabajos de revisión ni tampoco artículos cuyas poblaciones o muestras provenían de entornos educativos de los niveles inicial, primaria y secundaria, ya que de acuerdo con los criterios de 
la APA (2010b) pueden estar exentos del consentimiento informado siempre y cuando no genere ningún perjuicio a los participantes y se proteja su confidencialidad, ya sea que se trate de prácticas educativas o académicas que suelan ser cotidianas y en contextos educativos, cuestionarios anónimos, observaciones naturalistas, investigaciones de archivo, evaluación del desempeño o eficiencia laboral en entornos organizacionales, o cuando lo determine la Ley.

Para realizar la búsqueda, se revisó dentro del rubro 'procedimiento' de cada artículo, en el apartado Método, si cada una de las investigaciones objeto de estudio contenía la expresión 'consentimiento informado', 'consentimiento' o si se describía dicho proceso. En caso de no encontrar lo buscado, se revisaba si se refería una participación 'anónima y voluntaria'. De no encontrar la información requerida, se examinaba el rubro 'participantes', a fin de identificar si como criterio de inclusión de los participantes al estudio se consideraba el consentimiento informado o una participación anónima y voluntaria.

De otro lado, a fin de determinar si las revistas que contienen los artículos analizados establecen como norma la obtención del consentimiento informado o regulan el cumplimiento de los principios éticos, se procedió a revisar si las 'instrucciones para los autores' de cada revista indican que se ciñen a las normas APA, explicitan la obtención del consentimiento informado o exigen que la investigación postulada haya sido evaluada y aprobada previamente por un Comité de ética. En ese sentido, todas las revistas de las que se extrajeron los artículos analizados (47) contemplaron en sus criterios de publicación las normas de la APA; sin embargo, cinco de los ocho países contienen explícitamente el consentimiento informado en sus normas de publicación, con porcentajes entre $17 \%$ y $25 \%$, excepto Costa Rica (que lo presenta en una de sus dos revistas), y casi ningún país contiene como requisito, dentro de las normas para los autores, la evaluación de la investigación por algún Comité de ética, con excepción de Brasil que lo presenta en un $25 \%$ de sus revistas analizadas en este estudio (tabla 2).
Tabla 1

Artículos analizados publicados entre los años 1994 y 2012 en el catálogo de Latindex, por país

\begin{tabular}{lr}
\multicolumn{1}{c}{ País } & $F$ \\
\hline Perú & 259 \\
Colombia & 499 \\
Chile & 209 \\
México & 332 \\
Costa Rica & 21 \\
Argentina & 70 \\
Puerto Rico & 39 \\
Brasil & 316 \\
Bolivia & 48 \\
Paraguay & 75 \\
Uruguay & 33 \\
Venezuela & 23 \\
Total & 1924 \\
\hline
\end{tabular}

Tabla 2

Frecuencia de revistas que incluyen explícitamente el consentimiento informado y la evaluación del Comité de ética en sus normas de publicación, por país

\begin{tabular}{lccc}
\hline \multirow{3}{1}{ País } & \multicolumn{3}{c}{$\mathrm{f}$} \\
\cline { 2 - 4 } & Revistas & $\begin{array}{c}\text { Consentimiento } \\
\text { informado }\end{array}$ & Comité de ética \\
\hline Perú & 5 & $1(20 \%)$ & $0(0 \%)$ \\
Colombia & 9 & $2(22 \%)$ & $0(0 \%)$ \\
Chile & 6 & $1(17 \%)$ & $0(0 \%)$ \\
México & 10 & $0(0 \%)$ & $0(0 \%)$ \\
Costa Rica & 2 & $1(50 \%)$ & $0(0 \%)$ \\
Argentina & 4 & $0(0 \%)$ & $0(0 \%)$ \\
Puerto Rico & 1 & $0(0 \%)$ & $0(0 \%)$ \\
Brasil & 4 & $1(25 \%)$ & $1(25 \%)$ \\
Bolivia & 1 & $0(0 \%)$ & $0(0 \%)$ \\
Paraguay & 1 & $0(0 \%)$ & $0(0 \%)$ \\
Uruguay & 3 & $0(0 \%)$ & $0(0 \%)$ \\
Venezuela & 1 & $0(0 \%)$ & $0(0 \%)$ \\
Total & 47 & & \\
\hline & & & \\
\hline
\end{tabular}




\section{Resultados}

Las publicaciones en revistas de psicología de Argentina, Brasil, Chile, Colombia, Costa Rica, México, Paraguay, Perú, Uruguay y Venezuela reportan la obtención del consentimiento informado a partir del lapso transcurrido entre los años 2001 y 2009, con excepción de Puerto Rico, que presenta una publicación con consentimiento informado en el año 1995, y Bolivia, que no informa publicación alguna con consentimiento informado.

Argentina muestra cifras irregulares en la obtención del consentimiento informado. Sus frecuencias más elevadas se presentan en los años 2009, con nueve de trece publicaciones $(70 \%) ; 2011$, con tres de tres publicaciones (100\%); y 2012, con dos de tres publicaciones $(67 \%)$.

En Brasil, la frecuencia de las publicaciones con consentimiento informado ha sido de relativo incremento desde 1997 hasta el año 2011, en el que de 23 publicaciones, 16 informaron la consecución del consentimiento informado (70\%); sin embargo, en el año 2012 hubo una disminución, ya que de 39 publicaciones solo 17 informaron su obtención $(43 \%)$.

En Chile, en cambio, la consecución del consentimiento informado es irregular, siendo más elevado en los años 2007, con 10 de 18 publicaciones (56\%); 2008, con 12 de 23 publicaciones (52\%); 2011, con nueve de 17 publicaciones (53\%); y, 2012, con 18 de 36 publicaciones (50\%).

En Colombia, a pesar de no ser completamente regular la obtención del consentimiento informado conforme transcurren los años, tiende a un incremento desde el año 2009 hasta el año 2012, llegando a 36 publicaciones $(62 \%)$ con consentimiento informado de un total de 40 .

También Costa Rica presenta cifras irregulares de consentimiento informado en sus publicaciones. El reporte de su consecución disminuye en el año 2007 y luego, incrementa levemente en el año 2009, disminuye en el 2010 y, finalmente, en el año 2012, aumenta a tres de cinco publicaciones $(60 \%)$.

México presenta una tendencia de incremento de solicitar el consentimiento informado, siendo la frecuencia más elevada en el año 2008, con 9 de 25 publicaciones (36\%); luego, ocurre un descenso y un ligero ascenso en los años 2010, con 16 de 48 publicaciones (33\%); 2011, con 14 de 45 publicaciones (31\%); y 2012, con 22 de 69 publicaciones $(32 \%)$.

También en Paraguay la obtención es irregular, pues asciende a dos de cinco publicaciones (40\%) en el año 2008, disminuye en los siguientes años, luego aumenta en el año 2011 y se reduce nuevamente, llega a cinco publicaciones de 17 (29\%) al año 2012.

En Perú el reporte de consecución del consentimiento informado en las publicaciones es bajo e irregular, llega en el año 2012 a 8 de un total de 29 investigaciones $(28 \%)$.

Puerto Rico es el único país que presenta frecuencias elevadas de obtención del consentimiento informado en los años 1995, 1998 y 1999; sin embargo, las cifras disminuyen en el año 2002 y aumentan progresivamente, aunque no de modo regular. En el año 2012 llega a 9 de un total de 11 publicaciones $(82 \%)$.

En Uruguay y Venezuela, desde el año 2009, hay una tendencia de disminución de la consecución del consentimiento informado hasta el año 2011 y en el año 2012 hay un ligero incremento: 3 de 11 publicaciones (27\%) en Uruguay; y 2 de 11 publicaciones $(18 \%)$ en Venezuela.

Todos los países estudiados, con excepción de Puerto Rico y Brasil, presentan frecuencias más elevadas en sus primeras publicaciones sin la obtención del consentimiento informado. Además, Perú, Paraguay, Uruguay, Venezuela y Costa Rica optan con mayor anterioridad por el consentimiento informado que por la modalidad de investigaciones anónimas y voluntarias, mientras que Colombia, México y Brasil presentan primero investigaciones anónimas y voluntarias que con la modalidad de consentimiento informado. Chile, Argentina y Puerto Rico optan, con la misma frecuencia, por ambas modalidades en el mismo año (figura 1).

Por otro lado, se efectuó el análisis de las investigaciones experimentales publicadas, a pesar de no ser numerosas. Se encontró que entre los años 1994 y 2001 ninguna publicación de tipo experimental de los países estudiados obtuvo el consentimiento informado; sin embargo, en el año 2012 se aprecian frecuencias más elevadas (figura 2). 

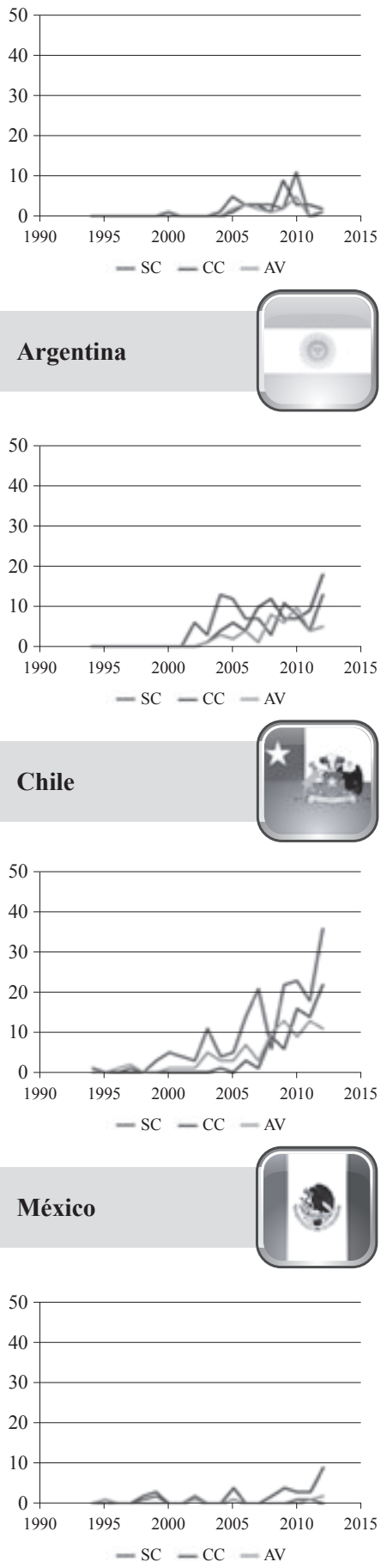

Puerto Rico

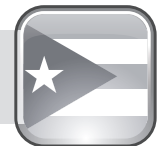

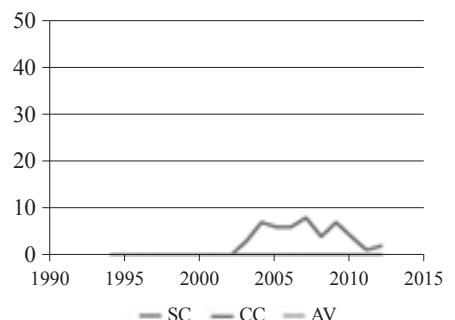
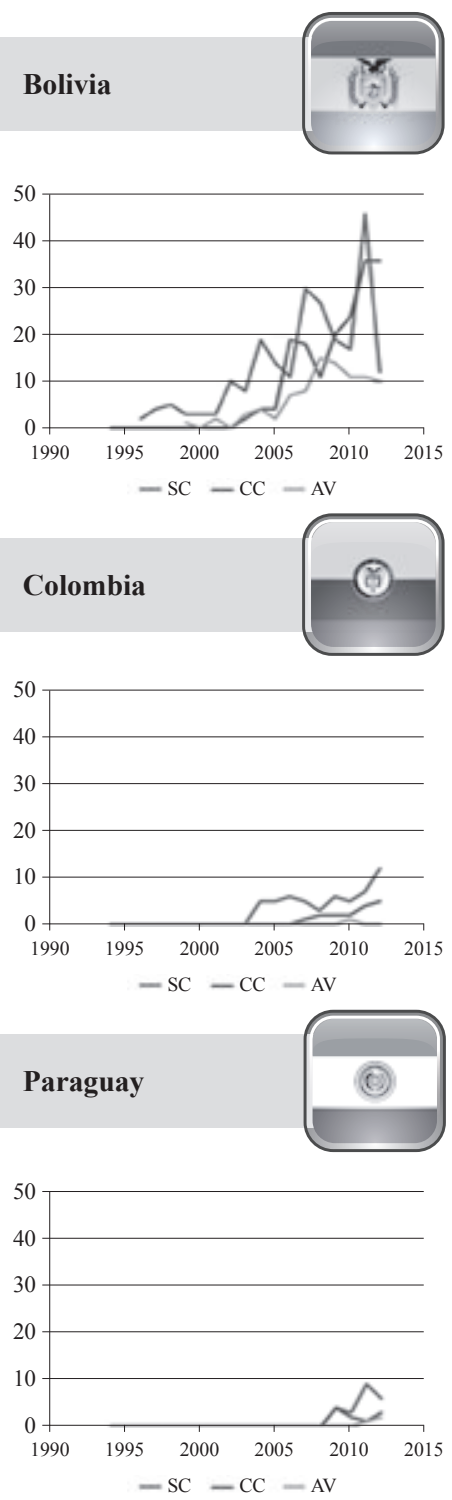

Uruguay

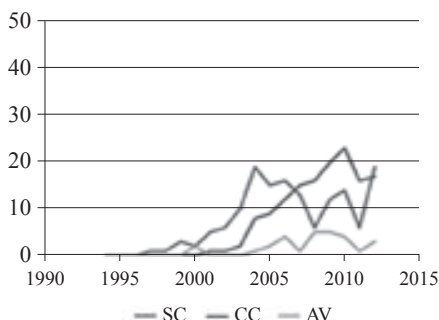

Brasil
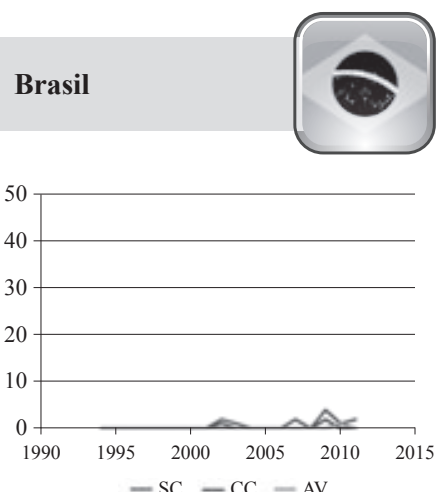

Costa Rica
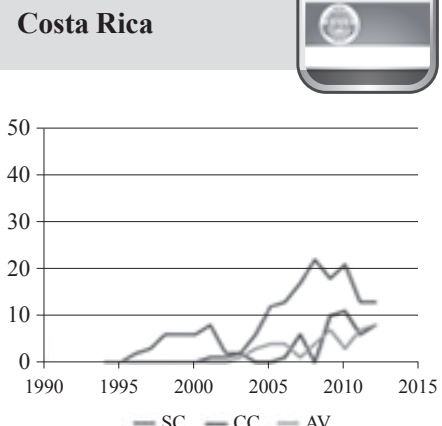

Perú

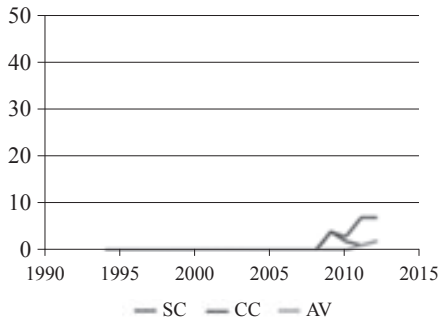

Venezuela

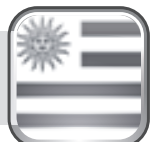

Figura 1. Consentimiento informado en artículos publicados en revistas latinoamericanas de psicología. Periodo 1994-2012 Nota: SC: sin consentimiento, CC: con consentimiento informado, AV: anónimo y voluntario. 
En Perú, a pesar de no mostrar una tendencia uniforme de incremento en la obtención del consentimiento informado de los estudios experimentales publicados durante los años 2000 al 2007, a partir del año 2008 ya se observa un aumento progresivo y en el año 2012, su única publicación, es con consentimiento informado.

En Colombia y Chile, las cifras que reportan la obtención del consentimiento informado en las publicaciones son fluctuantes, y en el año 2012 1legan a cinco de un total de siete publicaciones, en el caso de Colombia, y dos publicaciones para Chile, cifra que representa el total de sus publicaciones.

México muestra un progresivo aumento desde el año 2008, llega, en el año 2012, a tres de cinco publicaciones con consentimiento informado. Argentina también tiene un incremento hacia el año 2011, año de su última publicación experimental. Paraguay también presenta un aumento desde el 2007 hasta el 2008; sin embargo, en los años 2011 y 2012, ninguna de sus publicaciones reporta la obtención del consentimiento informado.

Costa Rica en sus dos únicas publicaciones experimentales en los años 2010 y 2011 no reporta la obtención del consentimiento informado. Tampoco lo informa Bolivia en sus publicaciones (en los años 2003, 2004, 2007 y 2009). Mientras que Puerto Rico y Brasil, en su única publicación experimental en los años 2010 y 2012, respectivamente, sí lo obtienen en ambos casos (figura 2).

\section{Discusión}

A pesar de que los hallazgos permiten vislumbrar que en algunos países la obtención del consentimiento informado es elevada, en términos generales, no hay un incremento progresivo y uniforme de la obtención del consentimiento informado en las publicaciones en revistas de psicología de los países latinoamericanos conforme transcurren los años y en el último año (2012) no se llega al cuartil superior. Estos reportes de bajo consentimiento informado coinciden con lo encontrado por Giraudeau, Caille, Gouge y Ravaud (2012) en su investigación efectuada sobre análisis de informes de ensayos aleatorizados en racimo y con el escaso manejo deontológico mostrado por los psicólogos argentinos en el estudio de Hermosilla (2006). Ello puede explicarse por lo encontrado por Sánchez (2010), quien afirma que la ética y la deontología profesional se constituyen en un déficit formativo.

Se están vulnerando los principios éticos de respeto, beneficencia y justicia en los participantes de las investigaciones, descritos en el Informe Belmont (1978), así como los principios de respeto a los derechos y dignidad e integridad, contemplados en el acuerdo de psicólogos de mercosur y países asociados (Hermosilla, 2000), sin avizorar (como señalan Ruiz \& Marijuán 2009) el deber como un ingrediente indispensable entre el saber y el proceder del profesional. También se está atentando contra los principios establecidos por la APA (2010a), a pesar de que todos los artículos publicados en las revistas estudiadas se basan estas normas, en cuyo manual de publicaciones se describe detalladamente el procedimiento de obtención de consentimiento informado y sus excepciones. Se tiende al abuso de la confianza atribuida al profesional que señala Eval (2012) o del altruismo de los usuarios o del que no sean conscientes que están siendo partícipes de una investigación (Vayena, Mastroianni \& Kahn, 2012).

A pesar de los reportes, los países de Puerto Rico, Argentina y Brasil son los que obtienen mejores resultados. Ello puede explicarse debido a que en los códigos de ética de los dos primeros países (Asociación de psicólogos de Puerto Rico, 2007; Federación de Psicólogos de la República Argentina, 1999) se norma la obtención del consentimiento informado. Sin embargo, en el caso de Brasil, a pesar de no explicitar la obtención del consentimiento informado en su código de ética (Conselho Federal de Psicologia, 2005), es el único país que reporta en las normas para autores de una de sus cuatro revistas analizadas, la evaluación del Comité de ética como requisito para postular los artículos a publicación. Al respecto, se acepta lo referido por Rodríguez (2005) quien señala que la conducta ética de la investigación está ligada al nivel de desarrollo del país, su contexto sociopolítico y cultural y, en ese sentido, de acuerdo con lo reportado por SCImago Journal \& Country Rank (1996-2012), Brasil domina la producción científica en América Latina, a pesar de que la visibilidad de las publicaciones latinoamericanas es baja 

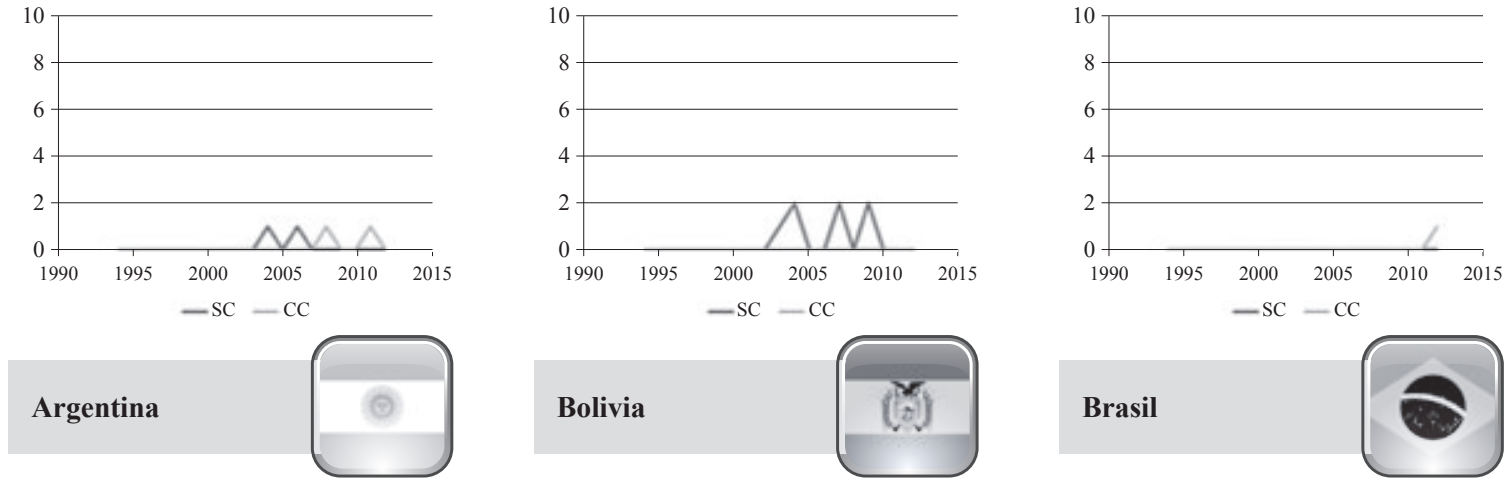

Brasil

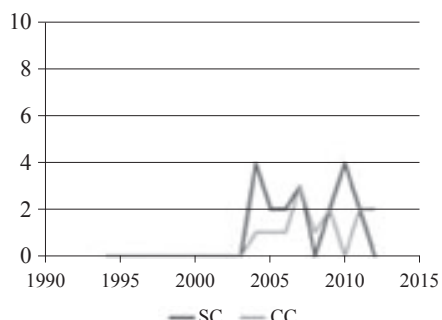

Bolivia
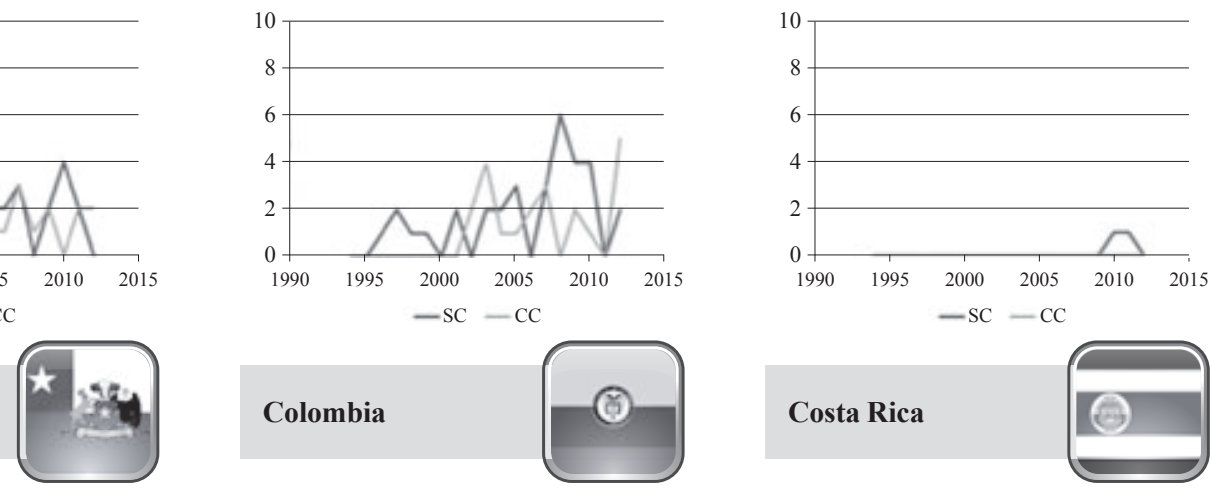

\section{Chile}

Colombia

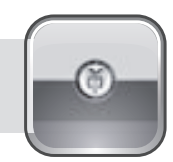

Costa Rica
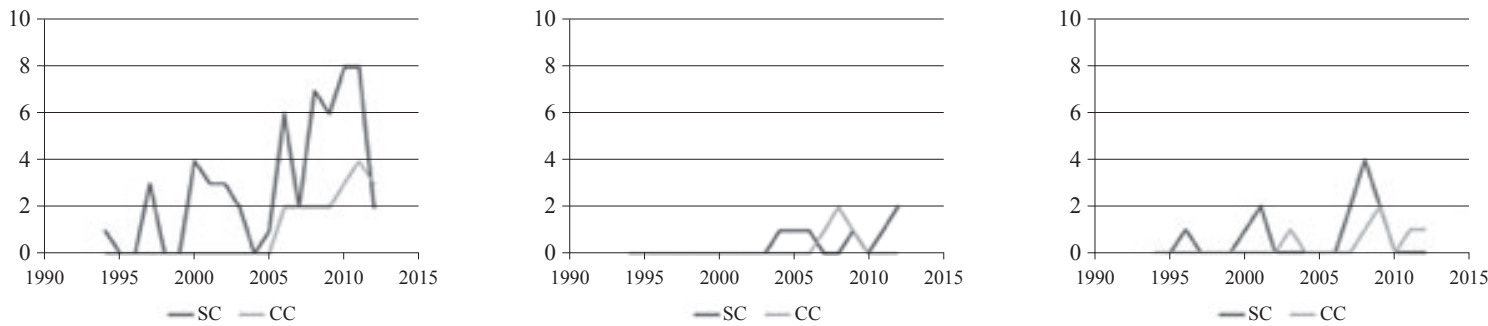

México

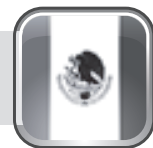

\section{Paraguay}

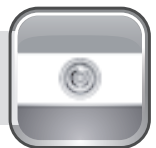

Perú
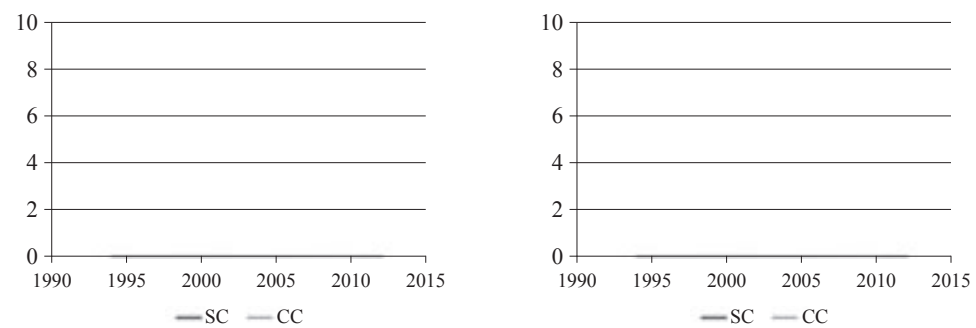

Uruguay

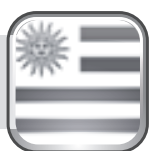

Venezuela

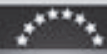

Figura 2. Consentimiento informado en investigaciones experimentales publicadas en revistas latinoamericanas de psicología. Periodo 1994-2012.

Nota: SC: sin consentimiento, CC: con consentimiento informado. 
en comparación con el promedio mundial y es el país latinoamericano, según la Organización de las Naciones Unidas para la Educación, la Ciencia y la Cultura (UNESCO, 2010), que más invierte en investigación. Por ello, se concuerda con Rama (2008), quien señala que el conocimiento es uno de los ejes centrales para el desarrollo de una región.

La experiencia de ser participante de experimentación es decisiva, motivo por el cual es necesario un discernimiento ético (Albornoz, Agüero, Cabrera \& Alonso, 2003; Carrasco, Rubio \& Fuentes, 2012), ya que se puede ejercer control sobre el participante (León, 2011; Ruiz \& Marijuán, 2009). En este sentido, aun cuando no son numerosos los estudios experimentales analizados, estos se discuten para tener una aproximación del estado actual de las consideraciones éticas en su realización.

En el año 2012, Brasil, Chile y Perú obtienen el consentimiento informado en todas sus investigaciones experimentales; Colombia y México lo consiguen para la mayoría de sus publicaciones, mientras que Paraguay no lo contempla en ninguno de sus experimentos. Por otro lado, Bolivia y Costa Rica no adquirieron el consentimiento informado en ninguna de sus publicaciones, que ocurrieron en los años anteriores, a diferencia de Argentina, que su primera investigación experimental fue sin consentimiento y en las dos siguientes si lo consideró.

En términos generales, para los estudios experimentales se estarían cumpliendo las condiciones para la obtención del consentimiento informado: voluntariedad, información, comprensión (Albornoz et al., 2003; Castorena, 2013) y razonamiento (Castorena, 2013). De ese modo, se estaría preservando el bienestar y la autonomía de los participantes, precisamente en un tipo de investigación en la que los riesgos de intervención -y no solo los beneficios-deben ser explicitados y considerados en la toma de decisión razonada y libre de los participantes de las investigaciones.

Las frecuencias alcanzadas de consentimiento informado en las publicaciones experimentales al año 2012 son más altas, comparadas con las de los años 2009 y 2010, lo que invita a permanecer alerta para no declinar en solicitar el consentimiento informado en la realización de investigaciones a publicarse en los años subsiguientes.
Esta realidad pone en evidencia la necesidad de promover la ética y deontología en la praxis psicológica desde las aulas universitarias, de tal manera que se eviten posibles daños a quienes participan de las investigaciones. En ese sentido, tanto las universidades, como el Colegio Profesional Nacional o los entes regionales tienen la obligación de enseñar o normar esta práctica ética.

Hace falta, como señalan Hermosilla et al. (2006), normar el consentimiento informado y además considerar la importancia de la formación deontológica del futuro profesional referida por Martínez et al. (2002), acompañada con su formación personal en las dimensiones ética y moral; además de la preparación deontológica de los docentes para su adecuado acompañamiento, de tal modo que el futuro profesional tenga un conocimiento claro y exhaustivo de sus deberes para ejercer su profesión.

Además, la conformación de los Comités de ética, que no ha sido considerada en el presente estudio, se constituye en un ente regulador de salvaguardar los principios éticos en la investigación científica.

Cabe referir que, al ser el valor máximo la dignidad humana, esta no puede estar desprovista de la veracidad y del respeto de participar de una investigación en la que el bienestar deberá garantizarse con el pleno uso de la razón de los participantes para decidir libremente si desean o no formar parte de los estudios empíricos.

Por ello, hace falta promover espacios de la reflexión y discusión crítica de la dimensión científica de la psicología desde un marco deontológico y ético.

Finalmente, posteriores estudios no solo podrán analizar reportes científicos sino también incluir la perspectiva de los investigadores y participantes, así como considerar la intervención de los Comités de ética en la evaluación de los trabajos de investigación.

\section{Referencias}

Acevedo, I. (2002). Aspectos éticos en la investigación científica, Ciencia y Enfermería, 8(1), 15-18.

Albornoz, C., Agüero, A., Cabrera, Y. \& Alonso, C. (2003). Aspectos éticos de la investigación 
clínica en seres humanos. Humanidades médicas, 3(2). Recuperado de http://scielo.sld.cu/scielo. php? script $=$ sci_arttext\&pid $=$ S1727-8120200300 0200003

Anaya, J. (2008). Consentimiento informado. Revista médica (Cochabamba), 19(29), 35-42. Recuperado de http://www.revistasbolivianas.org. bo/scielo.php?script $=$ sci_arttext $\&$ pid $=$ S207446092008002900007\&lng=en\&nrm=iso

American Psychological Association, APA (2002). Redline Comparison of APA Ethical Principles of Psychologists and Code of Conduct, December 1992 and December 2002. Recuperado de http:// www.apa.org/ethics/code/92-02 codecompare. pdfAmerican Psychological Association, APA (2010a). Ethical principles of psychologists and code of conduct. Washington: APA. Recuperado de http://www.apa.org/ethics/code/principles.pdf

American Psychological Association, APA (2010b). Publication manual of the American Psychological Association. (6 $6^{\mathrm{a}}$ ed.). Washington: APA.

Aréchiga, H. (2004). Los aspectos éticos de la ciencia moderna. En M. Aluja \& A. Birke (Coord.). El papel de la ética en la investigación científica y la educación superior (2a ed.). (pp. 41-66). México: FCE, Academia Mexicana de Ciencias. Recuperado de http://books.google.es/books?hl=es\&lr=\&id=A pD7RpdUOcC\&oi $=$ fnd \&pg $=$ PP $1 \& d q=\% 22 \% \mathrm{C}$ 3\%A9tica+e+investigaci $\% \mathrm{C} 3 \% \mathrm{~B} 3 \mathrm{n} \% 22$ \&ots= XkyhZpolTJ\&sig=5d81rJwE7VzZ1OQzbmZro Nbhlzc\#v $=$ onepage\&q $=\% 22 \% \mathrm{C} 3 \%$ A9tica $\% 20$ e\%20investigaci $\%$ C $3 \%$ B $3 n \% 22 \& f=$ false

Asociación de psicólogos de Puerto Rico. (2007). Código de ética. San Juan: Asociación de psicólogos de Puerto Rico. Recuperado de: http://psicologiacr.com/archivos/documentos/_Codigo_de Etica.pdf

Asociación Médica Mundial (2002). Declaración de Ginebra de la Asociación Médica Mundial. Navarra: Universidad de Navarra. Recuperado de http:// www.unav.es/cdb/ammginebra1.html

Bunge, M. \& Ardila, R. (2002). Filosofía de la Psicología. México D.F.: Siglo Veintiuno Editores.

Calva, R. (2006). Bioética. México: Mc Graw Hill.

Carrasco, P., Rubio, M. \& Fuentes, D. (2012). Consentimiento informado: un pilar de la investigación clínica, Aquichan, 12(1), 32-41.
Castorena, G. (2013). Consentimiento informado: un derecho fundamental en la relación médico paciente. Abogado Corporativo, 36, 27-30. Recuperado de http://works.bepress.com/guillermo_castorena/4

Ciccone, L. (2006). Bioética. Historia, principios, cuestiones Madrid: Palabra. Recuperado de http:// books.google.com.pe/books?id=wxrWiRsUI1M $\mathrm{C} \&$ printsec $=$ frontcover $\& \mathrm{dq}=\mathrm{bio} \% \mathrm{C} 3 \% \mathrm{~A} 9 \mathrm{tica} \&$ $\mathrm{hl}=\mathrm{es} \& \mathrm{sa}=\mathrm{X} \& \mathrm{ei}=19 \mathrm{a} 8 \mathrm{UKGBG}$ T69gTP0ICIDA $\&$ ved $=0$ CDMQ6wEwAA\# $\mathrm{v}=$ onepage $\& \mathrm{q}=\mathrm{bio} \%$ C3\%A9tica\&f=false

Chavarría, O. (2009). Declaración de Ginebra. Archivos de Pediatría del Uruguay, 80(1), 42. Recuperado de http://www.sup.org.uy/Archivos/adp80-1/pdf/ adp80-1_9.pdf

Colegio de Psicólogos de Chile. (1999). Código de ética profesional. Santiago de Chile: Colegio de Psicólogos de Chile Recuperado de http://www.colegiopsicologos.cl/wp-content/uploads/2009/08/ codigo-etica2.pdf

Colegio de Psicólogos de Cochabamba. (1995). Estatuto y Reglamento del Colegio de Psicólogos de Cochabamba. Cochabamba: Colegio de Psicólogos de Cochabamba. Recuperado de http://colpsicbba. blogspot.es/1347035206/estatuto-y-reglamentosdel-colegio-de-psicologos-de-cochabamba/\#

Colegio de Psicólogos de la Provincia de Santa Cruz. (s.a.). Código de Ética del Colegio de Psicólogos de la Provincia de Santa Cruz. Santa Cruz: Colegio de Psicólogos de la Provincia de Santa Cruz. Recuperado de http://www.google.com.pe/ url sa $=$ t\&rct $=\mathrm{j} \& \mathrm{q}=$ codigo $\% 20 \mathrm{de} \% 20$ etica $\% 20$ de $\% 20$ psicologos $\% 20$ de $\% 20$ santa $\% 20$ cruz\&sour ce $=$ web $\&$ cd $=5 \&$ cad $=$ rja\&ved $=0 \mathrm{CEgQFjAE \& url}$ $=\mathrm{http} \% 3 \mathrm{~A} \% 2 \mathrm{~F} \% 2 \mathrm{Fpsicologossantacruz}$. org. $\operatorname{ar} \% 2$ Fj\%2Fdocumentos\%2FCODIGO\%2520DE\%252 0ETICA $\% 2520$ CPPSC.doc\&ei=QwN2UZmIKO XT0wGnmoHQBQ\&usg=AFQjCNHGolT7JgxZ n0hQUq3cRpeas24hJg

Colegio de Psicólogos del Perú. (1981). Código de ética profesional del psicólogo peruano. Lima: Colegio de Psicólogos del Perú.

Colegio Profesional de Psicología de Costa Rica. (2010). Código de ética profesional.

Colombia, Congreso de la República. Ministerio de la Protección Social. Ley N 1090 de 2006. Código deontológico y bioético. 
Conselho Federal de Psicologia. (2005). Código de ética profissional do psicólogo. Brasília: Conselho Federal de Psicologia. Recuperado de http:/www. pol.org.br/pol/export/sites/default/pol/legislacao/ legislacaoDocumentos/codigo_etica.pdf

Coordinadora de Psicólogos del Uruguay, Sociedad de Psicología del Uruguay, Facultad de Psicología (UDELAR) y Facultad de Psicología (UCU) (2001). Código de ética profesional del psicólogo/a. Uruguay: Coordinadora de Psicólogos del Uruguay. Recuperado de http://psicologos. org.uy/codigo.html

Eval, N. (2012). Using informed consent to save trust. Journal of medical ethics, 0, 1-8. doi:10.1136/ medethics-2012-100490

Federación de Psicólogos de la República Argentina. (1999). Código de ética de la Federación de Psicólogos de la República Argentina. Buenos Aires: Federación de Psicólogos de la República Argentina. Recuperado de http://fepra.org.ar/docs/ Codigo_de_etica.pdf

Federación de Psicólogos de Venezuela (1981). Código de ética profesional del psicólogo. Caracas: Colegio de Psicólogos del distrito capital. Recuperado de http://fpv.org.ve/documentos/codigodeetica.pdf

Festinger, L. (1957). A theory of cognitive dissonance. Stanford, CA: Stanford University Press.

Figueroa, J. (1999). El significado del consentimiento informado dentro de los procesos de investigación social sobre reproducción. Medicina Perinatal y Reproducción Humana, 13, 32-42.

Flores, A. M. (2010). Situación de las revistas científicas en Iberoamérica. Visibilidad de las revistas. Módulo 1. Taller virtual de edición científica. Argentina: CAICYT

França-Tarragó, O. (1996). Ética para psicólogos. Introducción a la Psicoética. España: Desclée De Brouwer, S.A.

França, O., Añon, F., García, C., Grauert, R., Nuñez, J., Wodowooz, O. \& Queirolo, E. (1998). Ética en la investigación clínica. Una propuesta para prestarle la debida atención. Revista médica del Uruguay, 14, 192-201. Recuperado de http://www.rmu.org. uy/revista/1998v3/art3.pdf

Giraudeau, B., Caille, A., Gouge, A. \& Ravaud, P. (2012). Participant Informed Consent in Cluster Randomized Trials: Review. PLoS ONE, 7(7), p1-11. doi: 10.1371/journal.pone.0040436. Resumen recuperado de la base de datos EBSCO. (Acceso $\left.\mathrm{N}^{\mathrm{o}} 79784732\right)$

Hermosilla, A. M. (2000). Psicología y Mercosur: la dimensión ética de la integración y antecedentes del debate en Argentina. Fundamentos en Humanidades, 2, 63-76. Recuperado de http://dialnet. unirioja.es/servlet/articulo?codigo $=1280373$

Hermosilla, A. M., Liberatore, G., Losada, M., Della Savia, P. \& Zanatta, A. (2006). Dilemas éticos en el ejercicio de la psicología: resultados de una investigación. Fundamentos en Humanidades, Universidad Nacional de San Luis, 7(1-2), 91-106.

Informe Belmont (The National Comission for the protection of humans subjects of biomedical and behavioral research) (1978). Principios éticos y orientaciones para la protección de sujetos humanos en la experimentación. DHEW Publication No. (OS) 78-0012. Recuperado de http://www. unesco.org.uy/phi/aguaycultura/fileadmin/shs/ redbioetica/Informe_Belmont.pdf

International Union of Psychological Science (2008). Universal Declaration of Ethical Principles for Psychologists. Assembly in Berlin. Editado por International Union of Psychological Science. Recuperado de http://www.iupsys.net/about/governance/universal-declaration-of-ethical-principles-for-psychologists.html

Leibovich, A. (2000). La dimensión ética en la investigación psicológica. Investigaciones en Psicología, 5(1), 41-61.

León, F. J. (2011). Bioética. Madrid: Palabra. Recuperado de http://books.google.com.pe/books?id=B6B koRfKilwC\&printsec $=$ frontcover\&dq $=$ bio $\% \mathrm{C} 3 \%$ A9tica\&hl=es\&sa $=$ X\&ei=19a8UKGBG5T69gTP $0 \mathrm{ICIDA} \& v e d=0 \mathrm{CDYQ6wEwAQ} \# \mathrm{v}=$ onepage $\& \mathrm{q}$ $=$ bio $\% \mathrm{C} 3 \% \mathrm{~A} 9$ tica $\& \mathrm{f}=$ false

Lolas F. (2002). Bioética y medicina. Aspectos de una relación. Santiago de Chile: Editorial Biblioteca Americana.

Lucas, R. (2010). Explícame la bioética Madrid: Palabra. Recuperado de http://books.google.com.pe/bo oks? id=xbiEKNXZJzAC\&printsec $=$ frontcover $\& d$ $\mathrm{q}=$ bio $\% \mathrm{C} 3 \%$ A9tica\&hl $=$ es\&sa $=$ X\&ei $=\mathrm{CfC} 8 \mathrm{UIG}$ UGoea9gSd6YHABA\&ved=0CEIQ6wEwBTgK Mainetti, J. A. (1989). Ética médica. La Plata, Argentina: Quiron. 
Mann, T. (1994). Informed Consent for psychological research: Do subjects comprehed consent forms and undestand their legal rights?, Psychological Science, 5(3), 140-143.

Martínez, M., Buxarrais, M. R. \& Bara, F. (2002). La universidad como espacio de aprendizaje ético. Revista Iberoamericana de Educación, 2(29). Recuperado de http://www.rieoei.org/rie29a01.htm

McRae, A., Weijer, C., Binik, A. Grimshaw, J., Boruch,R., Brehaut, J., Donner, A., Eccles, M. Saginur, R., White, A. \& Taljaard, M. (2011). When is informed consent required in cluster randomized trials in health research? Trials, 12(202). doi: 10.1186/1745-6215-12-202. Recuperado de: http://www.ncbi.nlm.nih.gov/pmc/articles/PMC 3184061/

Milgram, S. (1963). Behavioral study of obedience. Journal of abnormal and social Psychology, 67(4), 371-378. Recuperado de http://isites.harvard.edu/ fs/docs/icb.topic606668.files/Course\%20Readings/94\%20The\%20Moral\%20Self/milgram. obedience.1963.pdf

Naciones Unidas. (s.a.). Historia de la redacción de la Declaración Universal de Derechos Humanos. Recuperado de http://www.un.org/es/documents/ udhr/history.shtml

Organización de las Naciones Unidas para la Educación, la Ciencia y la Cultura (UNESCO, 2010). Ranking latinoamericano de inversión, investigación, ciencia y tecnología. Recuperado de http://www. skyscraperlife.com/noticiasla/46778-ranking-latinoamericano-de-inversi $\% \mathrm{~F} 3 \mathrm{n}$-investigaci $\% \mathrm{~F} 3 \mathrm{n}$ ciencia-tecnologia-unesco.html

Pease-Carter, C. \& Minton, C. (2012). Counseling Programs' Informed Consent Practices: A Survey of Student Preferences. Counselor Education \& Supervision, 51(4), 308-319, doi: 10.1002/j.15566978.2012.00023.x. Resumen recuperado de la base de datos EBSCO. (Acceso No 84386713)

Potter, V. R. (1970). Bioethics: The Science of Survival. Perspectives in Biology and Medicine, 14, 127-153.

Rama, C. (2008). Tendencias de la educación superior en América Latina y el Caribe en el siglo XXI. Lima: Asamblea Nacional de Rectores.

Richaud, C. (2007). La ética en la investigación psicológica. Enfoques, 19(1-2), 5-18.
Rodríguez, E. (2005). Cultura ética e investigación en salud. Acta Bioethica, 9(1), 11-21. Recuperado de http://www.scielo.cl/pdf/abioeth/v11n1/art02.pdf

Ruiz, D. \& Marijuán, M. (2009). Bioética. Universidad del País Vasco (UPV-EHU): Especialidades Médico-Quirúrgicas. Área Medicina Legal y Forense. Recuperado de http://ocw2010.ehu.es/file.php/89/ bioetica/bioetica/Course_listing.html

Sánchez, M. J. (2010). Situación del área ética y deontología profesional en el proceso de formación académica de las carreras de la Facultad de Psicología (Tesis de Especialización en Docencia Universitaria, Universidad Nacional de La Plata, Argentina). Recuperado de http://sedici.unlp.edu.ar/bitstream/ handle/10915/19763/Documento_completo pdf? sequence $=1$

Sanz, R. (2008). La ética y los saberes de recursividad en la formación de psicólogos/as. Cuadernos de neuropsicología, 2(1), 24-31. Recuperado de https:// www.google.com.pe/url?sa=t\&rct=j\&q=\&esrc= s\&source $=$ web \&cd $=2 \& c a d=$ rja $\&$ sqi $=2 \& v e d=0$ CDYQFjAB\&url=http $\% 3 \mathrm{~A} \% 2 \mathrm{~F} \% 2 \mathrm{Fdialnet}$.unirioja.es\%2Fdescarga\%2Farticulo\%2F3997924. pdf\&ei=WQnVUNXWG-PB0AHa0ICIAQ\&us $\mathrm{g}=$ AFQjCNH0JTrtbbLf0feCC4D604ODA7gZt A\&bvm=bv.1355534169,d.eWU

SCImago Journal \& Country Rank (1996-2012). Country rankings. Recuperado de http://www.scimagojr.com/countryrank.php

Sociedad Mexicana de Psicología (2007). Código ético del psicólogo. México: Trillas.

Sociedad Paraguaya de Psicología (2012). Código de ética para el ejercicio profesional de la psicología en el Paraguay. Recuperado de https://docs. google.com/a/sopps.org/viewer? $\mathrm{a}=\mathrm{v} \&$ pid=sites\& srcid $=$ c29wcHMub3JnfHd3d3xneDo1OWVjYzB jZjIyZTY0MWVi

Supady, A., Antonie, V., Joachim, W., Udo, G. \& Northoff, G. (2011). How is informed consent related to emotions and empathy? An exploratory neuroethical investigation. Journal of medical ethics, 37(5), 311-7. doi:10.1136/jme.2010.037937. Resumen recuperado de la base de datos EBSCO. (Acceso No 60314870)

Tudor, M. (1939). An experimental study of the effect of evaluative labeling of speech fluency. (Thesis M.A., University of Iowa, United States). 
Unión Latinoamericana de Entidades de Psicología (2002). Declaración de principios. Puebla, México. Recuperado de http://www.ulapsi.org/espanhol/capa_2.php

Vayena, E., Mastroianni, A. \& Kahn, J. (2012). Ethical issues in health research with novel online sources. American Journal of Public Health, 102(12), 2225-2230. doi: 10.2105/AJPH.2012.300813. Resumen recuperado de la base de datos EBSCO. (Acceso $\mathrm{N}^{\circ} 83522164$ )

\section{Fecha de recepción: 25 de abril de 2013} fecha de aceptación: 9 de diciembre de 2013
Vélez, L.A. (1996). Ética médica: interrogantes acerca de la medicina, la vida y muerte. ( $2^{\mathrm{a}}$ ed.). Medellín: Corporación para investigaciones biológicas. Zimbardo, P., Haney, C., Curtis Banks, W. \& Jaffe, D. (1971). Stanford prison experiment: a simulation study of the psychology of imprisonment. Recuperado de http://dams.stanford.edu/depts/spc/uarch/ exhibits/spe/Narration.pdf 
